\title{
Compensation of the Impact of Disturbing Factors on Gas Sensor Characteristics
}

\author{
Zvezditza Nenova, Georgi Dimchev \\ Technical University of Gabrovo \\ Department of Electrical Engineering \\ 4, H. Dimitar Str., Gabrovo 5300, Bulgaria \\ nenova@tugab.bg; gdimchev@tugab.bg
}

\begin{abstract}
Methods for gas control have been extensively developed for the monitoring of air quality, for gas leak control, for the development of 'electronic nose' systems, etc. Metal oxide gas sensors have been widely used in particular. However, apart from changes in the controlled gas concentration, changes in their parameters also depend on ambient conditions. The main impact comes from temperature and humidity. Therefore, the compensation of these disturbances is important for increasing the accuracy of concentration measurements of the controlled gases and the reliability of control. The present paper proposes a method for compensating the impact of temperature and humidity on gas sensor characteristics using artificial neural networks. This compensation method is applied to the control of methane concentration by gas sensors TGS813 and TGS2611. The results obtained confirm the applicability of this method.
\end{abstract}

Keywords: compensation; gas sensors; disturbing factors; artificial neural networks

\section{Introduction}

Gas systems are widely used for monitoring outdoor and indoor air quality, in gas leak control systems, in the chemical industry, in the development and implementation of 'electronic nose' systems, etc. [1-5]. The control of air parameters is important for the protection of the environment and human health, as well as for providing safe working conditions. Gas pollution can spread over a wide area in a short time, and therefore methods and equipment for its measurement and monitoring are being extensively developed. A wide range of gas sensors [6-10] have been designed, including metal oxide gas sensors. Different kinds of metal oxides such as $\mathrm{SnO}_{2}, \mathrm{ZnO}, \mathrm{Fe}_{2} \mathrm{O}_{3}, \mathrm{WO}_{3}, \mathrm{Co}_{3} \mathrm{O}_{4}$, etc. [1116] are used as sensing materials. Their operating principle is based on increasing the conductivity of the surface film of the sensitive element when the test gas is adsorbed. Depending on the composition of the surface film, the sensor responds 
to different gases such as carbon monoxide, carbon dioxide, ethanol, methane, propane, ammonia, hydrogen sulfide, hydrogen, etc. [6-11]. Metal oxide gas sensors have high sensitivity, low cost and a short response time. However, their characteristics are influenced by various ambient parameters which act as disturbing factors in gas control. Temperature and humidity have a major impact among these factors [7-10, 17, 18]. To enhance the measurement accuracy and reliability of control, compensating the impact of disturbing factors on gas sensors is of prime importance.

A method for compensating the impact of ambient temperature and humidity on gas sensor characteristics by using artificial neuron networks (ANN) is proposed in this paper. The method is based on a three-dimensional approximation of the gas sensor characteristics employing an ANN. The method is applied to the control of methane concentration with gas sensors TGS813 and TGS2611, and the results of that implementation are shown.

\section{ANN Compensation Method}

For metal oxide gas sensors, the input quantity is the unknown concentration, Conc, of the gas being controlled, which leads to a change in the output quantity of the sensor - its resistance, $R s$. The ambient factors, temperature, $t$, and relative humidity, $R H$, which act as disturbing factors, also have an effect on this resistance (Fig. 1).

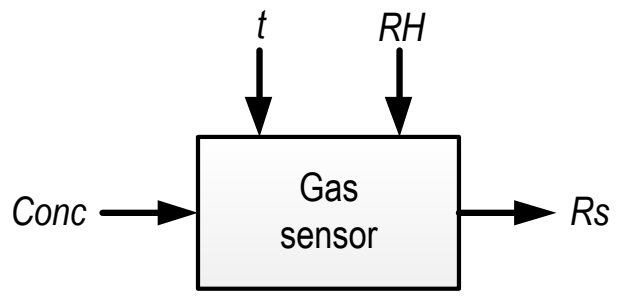

Figure 1

Input quantity Conc and disturbing factors $t$ and $R H$ for gas sensors

Sensor manufacturers usually report gas sensor characteristics as the sensor resistance ratio, $R s / R o$, under various gas concentrations and ambient conditions, i.e.:

$\left(\frac{R s}{R o}\right)=f($ Conc $, t, R H)$,

where $R s$ is sensor resistance, and $R o$ is resistance for referent concentration, temperature and humidity. 
However, these characteristics are usually given only for some values of the disturbing factors

$$
\begin{array}{r}
\left(\frac{R s}{R o}\right)_{i}=f(\text { Conc }) \mid t_{i}, R H_{i}=\text { const } \\
i=1,2, \ldots, n .
\end{array}
$$

It should be noted that in practice it is difficult to calibrate the gas sensor, and the impact of disturbing factors is usually given only for characteristics at a fixed concentration.

$$
\begin{gathered}
\left(\frac{R s}{R o}\right)_{i}=f\left(t_{i}, R H_{i}\right) \mid \text { Conc }=\text { const } \\
i=1,2, \ldots, n .
\end{gathered}
$$

If in the application of gas sensors the operating characteristic is chosen for fixed $t$ and $R H$ (most commonly at $20^{\circ} \mathrm{C} / 65 \% \mathrm{RH}$ ), this inevitably leads to measurement errors due to changes in ambience. In order to take into consideration the impact of $t$ and $R H$, it is necessary to approximate the transformation function of the sensor and use it in applications.

Based on equation (1), this should be a three-dimensional approximation. Difficulties arise owing to the great nonlinearity of characteristics (1) - (3). Additionally, as was mentioned, the sensor characteristics usually cannot be given at uniform points that can be used in function approximation.

A theoretical method for polynomial approximation of a multivariable sensor characteristic was proposed in [18]. In its practical application only the compensation of the impact of humidity, $R H$, on gas sensor characteristics is shown. However, introducing a second disturbing factor (such as temperature), would substantially increase the number of equations and coefficients used.

Artificial neural networks can also be employed for solving different problems with many input parameters. It is very common to use ANN for gas and odor recognition, the classification of products, the control of environmental parameters, etc. [19-23]. In [24] an ANN-based virtual compensator for correcting the effect of a disturbing variable in transducers is proposed. That method is applied to a strain-gauge transducer-based pressure measurement system. The correction is carried out by a nonlinear two-dimensional artificial neural networkbased inverse model of the transducer. ANN has also been used for twodimensional approximation of humidity sensor characteristics in order to compensate for the impact of one factor - temperature [25]. This approach is shown to achieve the highest accuracy for a nonlinear transformation function compared to polynomial and interpolation methods. The compensation for temperature effects in gas sensors via ANN is reported in [26]. 
The ANN-based method proposed in this paper aims to compensate for the impact on gas sensors of both ambient temperature and humidity through a threedimensional approximation of the gas sensor characteristics.

The method is implemented in two stages: training of the ANN, and real measurement and control of gas concentration.

In the training stage, the calibration characteristics given by the manufacturers are used. Input parameters for the ANN are: the gas sensor resistance ratio, Rs / Ro, ambient temperature, $t$, and relative humidity, $R H$, and an output parameter - the concentration, Conc, of the respective gas. The points of training can be complemented, whenever possible, by a functional approximation of characteristics (2) and offsets based on (3).

As a result of the ANN training, a three-dimensional approximation of the sensor characteristics is performed with relationships of the type

Conc $=f(R s / R o, t, R H, W, a, b)$,

where $W, a$ and $b$ are ANN parameters.

In the stage of real measurement and control, in addition to measuring the gas sensor parameter, it is necessary to measure temperature separately by means of a temperature sensor and air humidity by means of a humidity sensor. A schematic diagram of the method implementation for one gas is shown in Fig. 2.

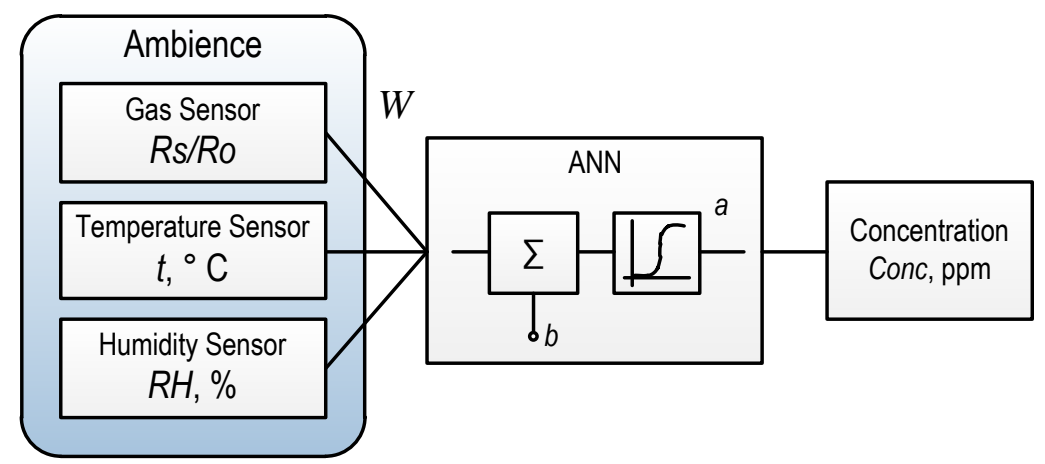

Figure 2

Schematic diagram of the implementation of the ANN compensation method for one gas

On the basis of the approximation relationships obtained (equation (4)) the measured gas concentration is determined. The changes in ambient temperature and humidity are taken into account, and therefore, their impact on gas sensor characteristics is compensated for.

The method can also be employed for a higher order approximation for a greater number of disturbing factors. 


\section{Results and Discussion}

The method is applied to compensate for the impact of temperature and humidity on gas sensors TGS813 and TGS2611 for the control of methane concentration. Sensor characteristics (2) and (3) at $1000 \mathrm{ppm}$ and $5000 \mathrm{ppm}$, respectively, given by manufacturers have been used [7].

For sensor TGS813, Ro is the gas sensor resistance for the referent concentration of $1000 \mathrm{ppm}$ and $20^{\circ} \mathrm{C} / 65 \% \mathrm{RH}$. According to experimental characteristics [7], in logarithmic scale, the characteristics $R s / R o=f($ Conc) of the sensor for given $t$ and $R H$ are straight lines and can be represented by an equation of the form

$y=a_{0}+a_{1} \cdot x$,

where $y=\lg ($ Rs / Ro $), x=\lg ($ Conc $)$.

These characteristics are parallel straight lines; i.e., coefficient $a_{1}$ is constant and can be determined by any of the experimental relationship $R s / R o=f($ Conc $)$ for $t=$ const and $R H=$ const .

Variations in temperature and relative humidity lead only to a change in the offset $a_{0}$ of these characteristics. This offset has been calculated on the basis of characteristics (3) at the reference concentration for temperature variation in the range of $-10^{\circ} \mathrm{C} \ldots+40^{\circ} \mathrm{C}$ and relative humidity in the range of $0 \ldots 100 \% \mathrm{RH}$ [7].

Thus, the family of characteristics are obtained analytically at various temperatures in the range of $-10^{\circ} \mathrm{C} \ldots+40^{\circ} \mathrm{C}$ and fixed humidities of $0,20,40,65$ and $100 \%$ RH. Fig. 3 presents this family of characteristics for sensor TGS813 at $65 \% \mathrm{RH}$, showing the impact of temperature.

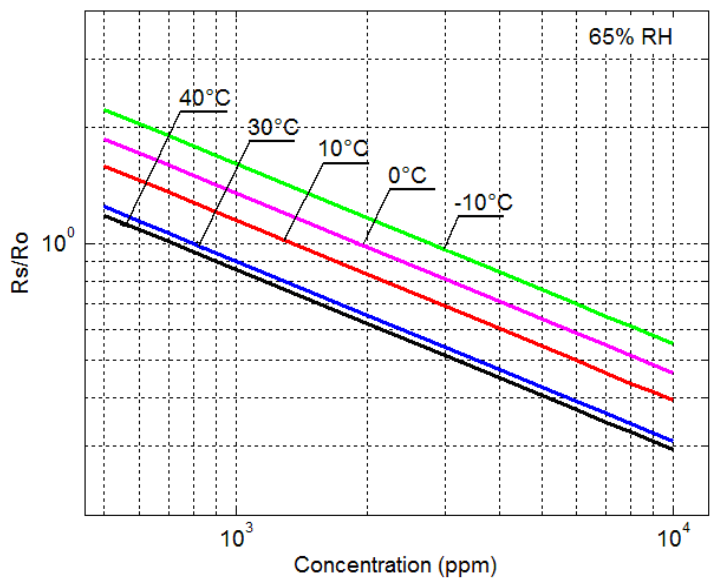

Figure 3

Analytically obtained characteristics for sensor TGS813 for temperature variation and 65\% RH 
Similarly, based on the experimental characteristics [7] for sensor TGS2611, the families of characteristics have been obtained at temperatures in the range of $10^{\circ} \mathrm{C}$ to $40^{\circ} \mathrm{C}$ and for fixed values of relative humidity of $35,50,65$ and $95 \% \mathrm{RH}$. For this sensor, $R o$ is the resistance at a referent concentration of $5000 \mathrm{ppm}$ and $20^{\circ} \mathrm{C} / 65 \%$ RH. Fig. 4 presents the family of analytically obtained characteristics for sensor TGS2611 for temperature variation and $65 \% \mathrm{RH}$.

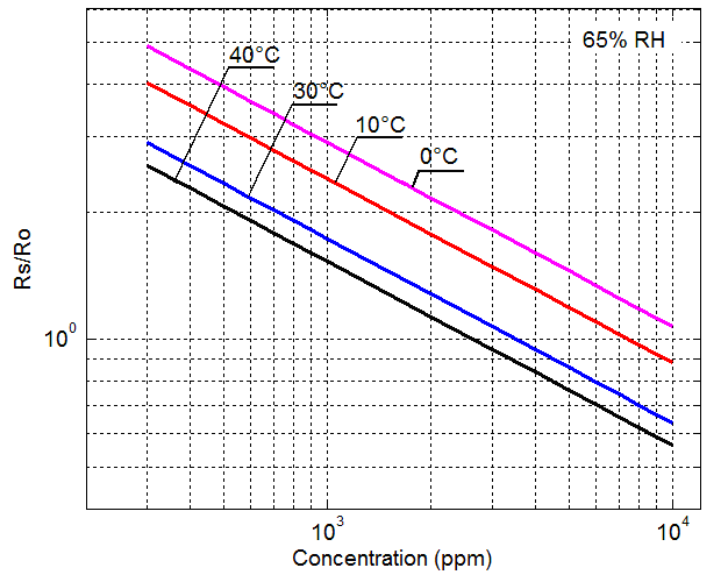

Figure 4

Analytically obtained characteristics for sensor TGS2611 for temperature variation and $65 \% \mathrm{RH}$

The experimental characteristics, apart from those at $20^{\circ} \mathrm{C} / 65 \% \mathrm{RH}$, and the whole set of analytically obtained characteristics for each sensor TGS813 and TGS2611 is used for ANN training. The experimental characteristics at $20^{\circ} \mathrm{C} / 65 \% \mathrm{RH}$ are used for checking the accuracy of the proposed method.

Experiments with various algorithms have been carried out for the ANN training. The best convergence for the smallest number of neurons is obtained in training with the LM algorithm (Levenberg-Marquardt back propagation). The obtained ANN with back propagation of error has three layers: two hidden (input, intermediate) and one output layer. The first layer consists of three neurons, one for each input quantity, the second layer is made up of seven neurons, and the third layer has one neuron (Fig. 5).

In both the first and second layers the transfer functions of neurons $\left(f^{1}\right)$ and $\left(f^{2}\right)$ are sigmoidal, and in the third layer $\left(f^{3}\right)$ it is linear.

The neural network has the following form

$Y=f^{3}\left(L W^{3,2} f^{2}\left(L W^{2,1} f\left(I W^{1,1} p+b^{1}\right)+b^{2}\right)+b^{3}\right)$,

where $Y=$ Conc $, p_{1}=R s / R o, p_{2}=t, p_{3}=R H$. 


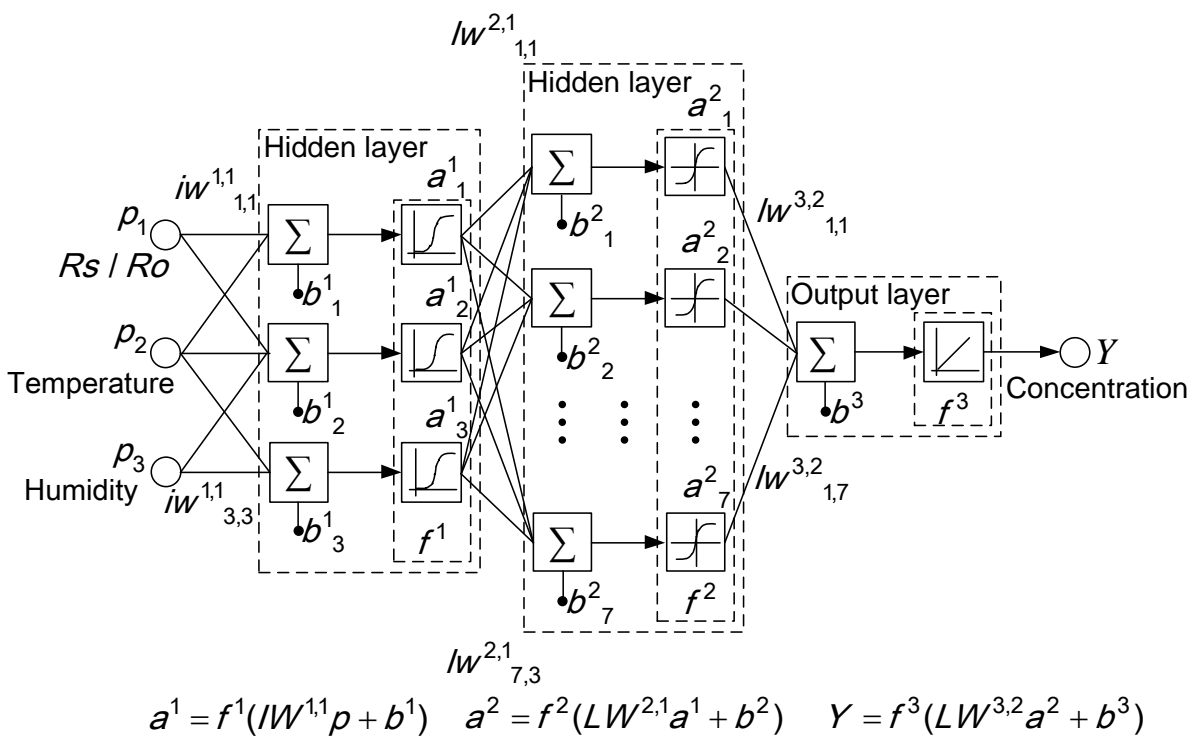

Figure 5

ANN for approximating the gas sensor characteristics

Fig. 6 shows the results from the output of trained neural networks for sensors TGS813 and TGS2611 and surfaces with different humidity levels are illustrated.

Thus, on the basis of three-dimensional approximation of sensor characteristics resulting from ANN training, the value of methane concentration can be obtained when the impact of temperature and relative humidity is compensated for.

Fig. 7 shows the characteristics obtained by ANN, illustrating the joint impact of ambient temperature and humidity on the sensors resistance ratio, $R s / R o$, at a concentration of 1000ppm for sensor TGS813 and 5000ppm for sensor TGS2611. 


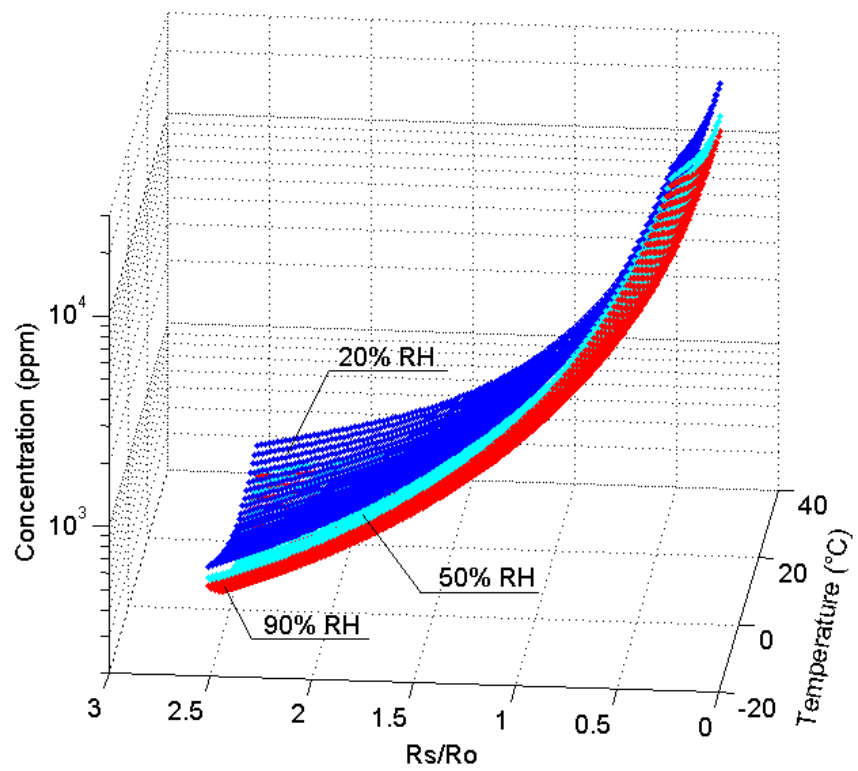

a

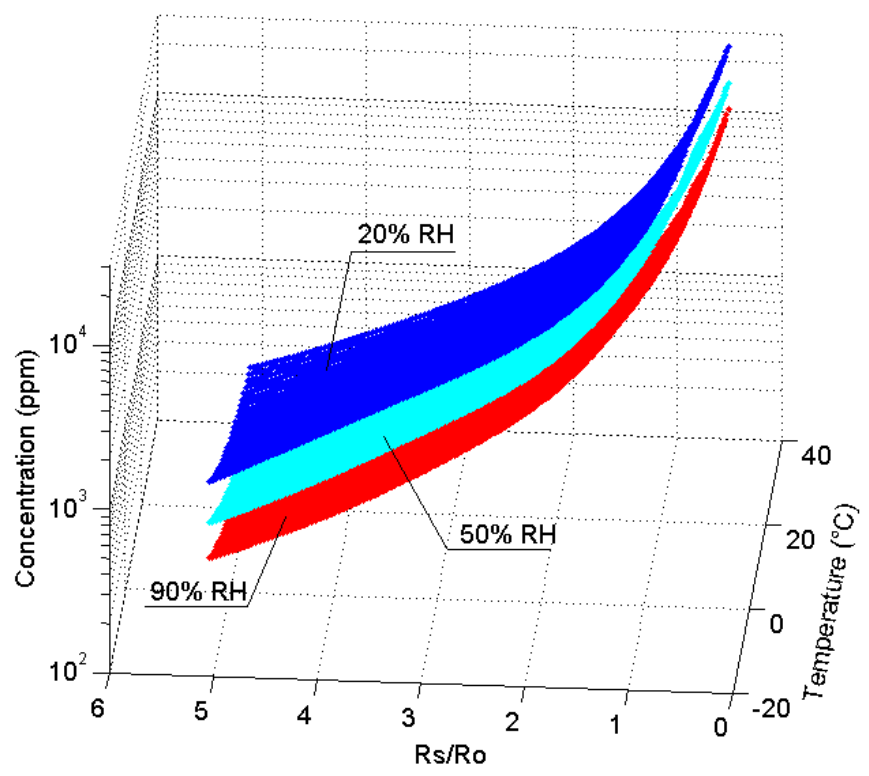

b

Figure 6

Results from the output of the trained neural network: a) for sensor TGS813; b) for sensor TGS2611 


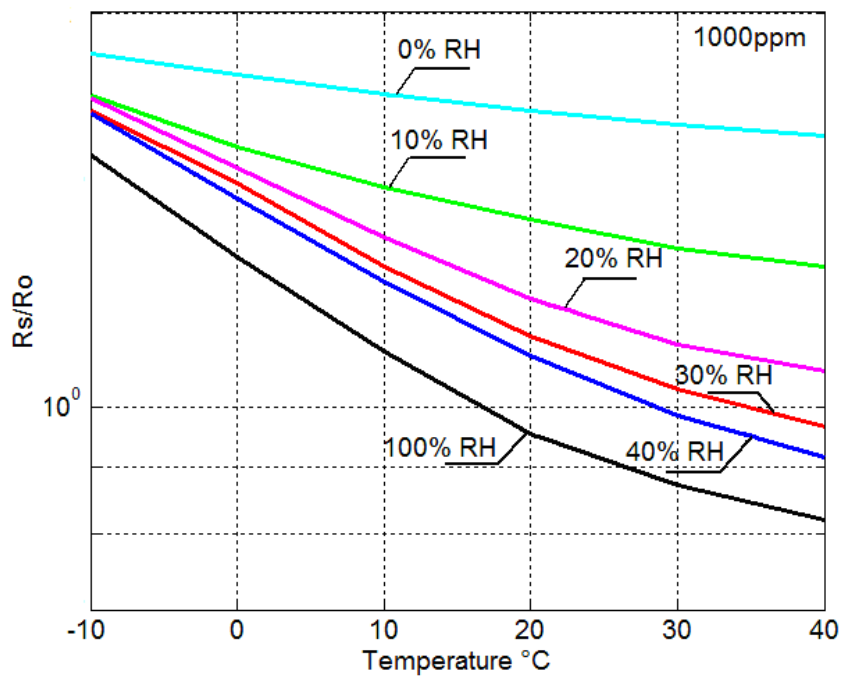

a

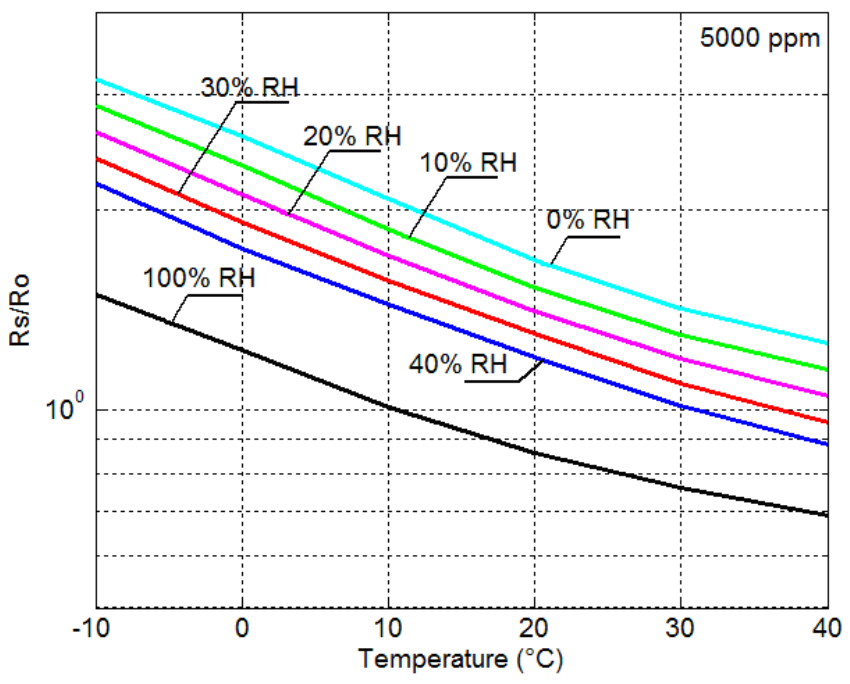

b

Figure 7

Impact of ambient temperature and humidity on sensors resistance ratio Rs/Ro: a) of sensor TGS813 at referent concentration of 1000ppm; b) of sensor TGS2611 at referent concentration of 5000ppm 
The algorithm for compensating for the impact of temperature and humidity on gas sensors readings by means of ANN in the process of gas control is shown in Fig. 8 .

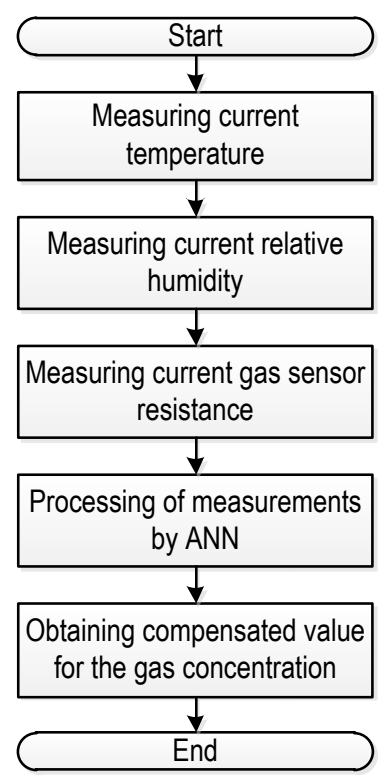

Figure 8

ANN compensation algorithm in gas sensors

To estimate the error which occurs if there is no compensation for temperature and humidity on sensor characteristics, the absolute error

$$
\Delta \text { Conc }_{t, R H}=\text { Conc }- \text { Conc }_{t, R H}
$$

and normalized error

$$
\varepsilon_{n t, R H}=\frac{\Delta \text { Conc }_{t, R H}}{\text { Conc }_{\max }-\text { Conc }_{\min }} .100 \%,
$$

are calculated, where Conc is the concentration based on the operating characteristic without compensation; $\operatorname{Conc}_{t, R H}$ is the real concentration corresponding to characteristics given the variation in temperature and relative humidity; and Conc $_{\max }-$ Conc $_{\min }$ is the range of concentration variation for each sensor.

The errors occurring for 1000ppm and $2000 \mathrm{ppm}$ when using the basic characteristics of sensors at $20^{\circ} \mathrm{C} / 65 \% \mathrm{RH}$, without taking into account the variation in temperature and in relative humidity, are shown in Tables 1 and 2 respectively. 
Table 1

Normalized error for sensor TGS813 without compensation when using the basic characteristic at $20^{\circ} \mathrm{C} / 65 \% \mathrm{RH}$

\begin{tabular}{|c|c|c|}
\hline$t^{\circ} \mathrm{C} / \% \mathrm{RH}$ & $\begin{array}{c}\text { Conc, } \\
\mathrm{ppm}\end{array}$ & $\begin{array}{c}\varepsilon_{n} t, R H \\
\%\end{array}$ \\
\hline$-10^{\circ} \mathrm{C} / 0 \% \mathrm{RH}$ & 1000 & -28.7 \\
\hline$-10^{\circ} \mathrm{C} / 0 \% \mathrm{RH}$ & 2000 & -60.0 \\
\hline $40^{\circ} \mathrm{C} / 100 \% \mathrm{RH}$ & 1000 & 3.7 \\
\hline $40^{\circ} \mathrm{C} / 100 \% \mathrm{RH}$ & 2000 & 7.3 \\
\hline
\end{tabular}

Table 2

Normalized error for sensor TGS2611 without compensation when using the basic characteristic at $20^{\circ} \mathrm{C} / 65 \% \mathrm{RH}$

\begin{tabular}{|c|c|c|}
\hline$t^{\circ} \mathrm{C} / \% \mathrm{RH}$ & $\begin{array}{c}\text { Conc, } \\
\mathrm{ppm}\end{array}$ & $\begin{array}{c}\varepsilon_{n} t, R H, \\
\%\end{array}$ \\
\hline$-10^{\circ} \mathrm{C} / 0 \% \mathrm{RH}$ & 1000 & -16.5 \\
\hline$-10^{\circ} \mathrm{C} / 0 \% \mathrm{RH}$ & 2000 & -32.5 \\
\hline $40^{\circ} \mathrm{C} / 100 \% \mathrm{RH}$ & 1000 & 5.9 \\
\hline $40^{\circ} \mathrm{C} / 100 \% \mathrm{RH}$ & 2000 & 11.9 \\
\hline
\end{tabular}

These results confirm the necessity of compensating for the impact of temperature and relative humidity.

Using the trained neural network, the values Conc $_{A N N}$ of methane concentration have been obtained at various values of resistance, temperature and humidity. The absolute error is determined based on these values

$\triangle$ Conc $=$ Conc $_{A N N}-$ Conc

and the normalized error of the ANN method is

$\varepsilon_{n}=\frac{\Delta \text { Conc }}{\text { Conc }_{\max }-\text { Conc }_{\min }} \cdot 100 \%$,

where Conc $_{A N N}$ is the concentration value, determined using the trained neural network; Conc is the respective real concentration value from the basic experimental characteristics which have not taken part in training; and $C o n c_{\max }-$ Conc $_{\min }$ is the range of concentration variation for each sensor.

The experimental basic characteristics, which have not been used in training, and those obtained by ANN for the two sensors are shown in Fig. 9.

Fig. 10 gives a graphic presentation of normalized errors (equation (10)) when employing the proposed method for compensation by ANN. 


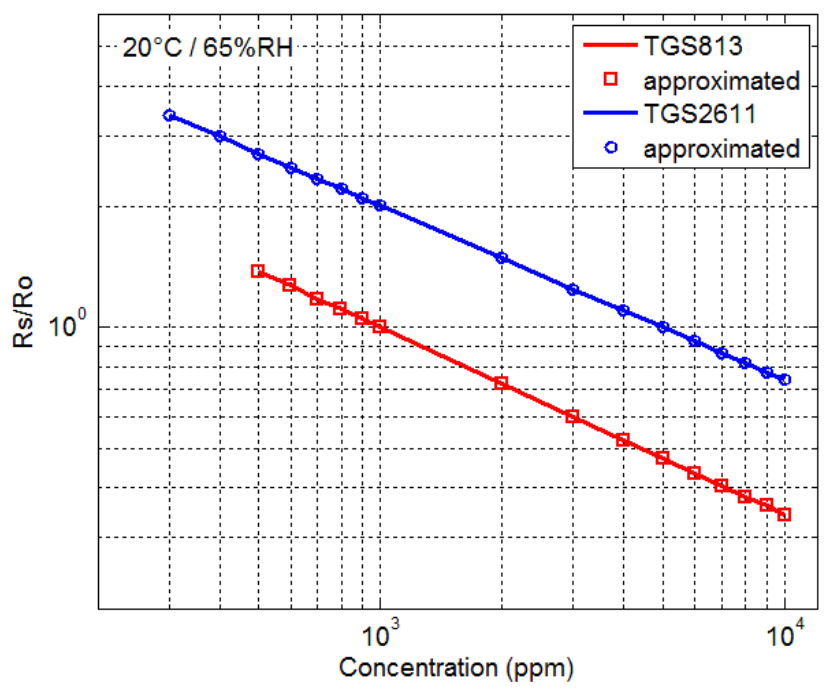

Figure 9

Experimental basic characteristics and characteristics obtained by ANN

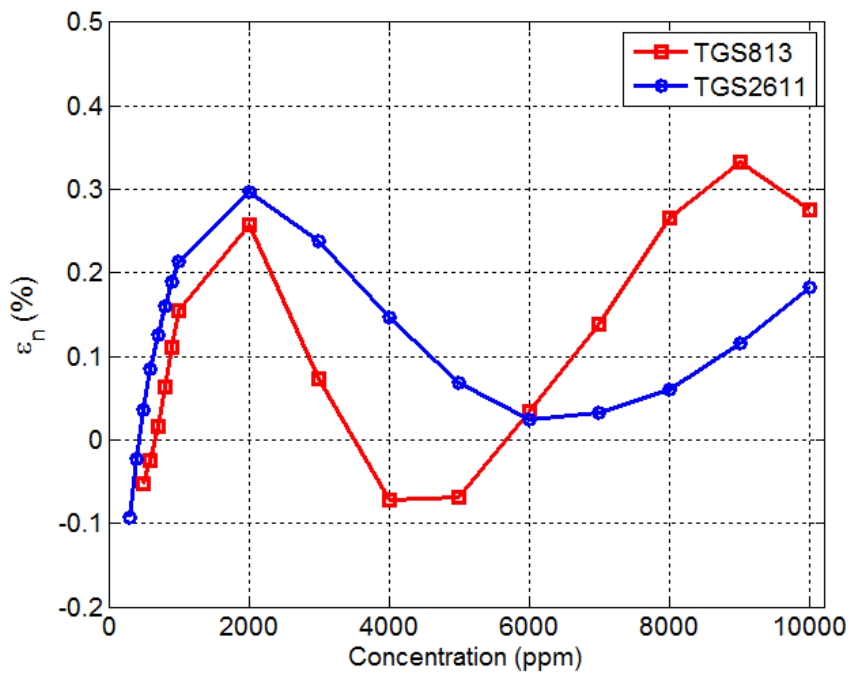

Figure 10

Normalized errors when implementing the ANN-compensation method

The obtained results show that the normalized error of the ANN method is in the range of $-0.05 \%$ to $+0.35 \%$ for sensor TGS 813 , and $-0.1 \%$ to $+0.3 \%$ for sensor TGS2611, which confirms the effectiveness of the implementation of the proposed ANN compensation method. 


\section{Conclusions}

On the basis of the research conducted, the following conclusions can be drawn:

- ambient temperature and humidity have a substantial impact on metal oxide gas sensor characteristics;

- a method is proposed for compensating for the impact of temperature and humidity on gas sensors by using ANN for three-dimensional approximation of their characteristics;

- to compensate for the impact of ambient conditions on gas sensors of type TGS813 and TGS261 for the measurement and control of methane, a trained ANN with back propagation of error with two hidden (input and intermediate) and one output layers has been obtained;

- the three-dimensional approximation of gas sensor characteristics by the trained neural network allows the impact of temperature and humidity to be compensated for and the normalized error is from $-0.05 \%$ to $+0.35 \%$ for sensor TGS 813 and from $-0.1 \%$ to $+0.3 \%$ for sensor TGS2611.

- the proposed method of compensating for the impact of disturbing factors by ANN can also be used for other types of sensors, as well as for performing higher order approximation with a greater number of disturbing factors.

\section{References}

[1] G. F. Fine, L. M. Cavanagh, A. Afonja and R. Binions: Metal Oxide SemiConductor Gas Sensors in Environmental Monitoring, Sensors 2010, 10, pp. 5469-5502

[2] M. Fleischer, M. Lehmann: Solid State Gas Sensors - Industrial Application, Springer-Verlag Berlin Heidelberg, 2012, p. 269

[3] K. Arshak, E. Moore, G. M. Lyons, J. Harris and S. Clifford: A Review of Gas Sensors Employed in Electronic Nose Applications, Sensor Review, Vol. 24, Number 2, 2004, pp. 181-198

[4] A. D. Wilson and M. Baietto: Applications and Advances in ElectronicNose Technologies, Sensors, 2009, 9, pp. 5099-5148

[5] S. Zampolli, I. Elmi, F. Ahmed1, M. Passini, G. C. Cardinali, S. Nicoletti, L. Dori: An Electronic Nose Based on Solid State Sensor Arrays for LowCost Indoor Air Quality Monitoring Applications, Sensors and Actuators B 101, 2004, pp. 39-46

[6] T. Nenov, P. Panteleev: Gas Sensors for Environmental Monitoring. Automatica\&Informatics, 2010, No. 1, pp. 16-19

[7] FIGARO Engineering Inc. Products - Gas Sensors (www.figaro.co.jp/en/product/) 
[8] SYNKERA Technologies Inc. Products (www.synkera.com)

[9] e2v Technologies. Products (www.e2v.com)

[10] Sencera. Products (www.sencera.com)

[11] N. Barsan and U. Weimar: Understanding the Fundamental Principles of Metal Oxide-based Gas Sensors; the Example of CO Sensing with $\mathrm{SnO}_{2}$ Sensors in the Presence of Humidity, J. Phys.: Condens. Matter, 2003, 15, pp. 813-839

[12] K. Shimizu, I. Chinzei, H. Nishiyama, S. Kakimoto, S. Sugaya, W. Matsutani, A. Satsuma: Doped-Vanadium Oxides as Sensing Materials for High Temperature Operative Selective Ammonia Gas Sensors. Sensors and Actuators B 141, 2009, pp. 410-416

[13] N. Han, L. Chai, Q. Wang, Y. Tian, P. Deng, Y. Chen: Evaluating the Doping Effect of Fe, $\mathrm{Ti}$ and $\mathrm{Sn}$ on Gas Sensing Property of $\mathrm{ZnO}$, Sensors and Actuators B 147, 2010, pp. 525-530

[14] Ch.-Y. Lin, Y.-Y. Fang, Ch.-W. Lin, J. J. Tunney, K.-Ch. Ho: Fabrication of $\mathrm{NO}_{\mathrm{x}}$ Gas Sensors Using $\mathrm{In}_{2} \mathrm{O}_{3}-\mathrm{ZnO}$ Composite Films, Sensors and Actuators B 146, 2010, pp. 28-34

[15] Z. Jiang, Z. Guo, B. Sun, Y. Jia, M. Li, J. Liu: Highly Sensitive and Selective Butanone Sensors Based on Cerium-doped $\mathrm{SnO}_{2}$ Thin Films, Sensors and Actuators B 145, 2010, pp. 667-673

[16] G. Korotcenkov, B. K. Cho: Thin Film $\mathrm{SnO}_{2}$-based Gas Sensors: Film Thickness Influence, Sensors and Actuators B 142, 2009, pp. 321-330

[17] Ch. Wang, L. Yin, L. Zhang, D. Xiang and R. Gao: Metal Oxide Gas Sensors: Sensitivity and Influencing Factors, Sensors 2010, 10, pp. 20882106

[18] J. Janiczek: Approximation of a Multivariable Sensor Characteristic Applied to the Carbon Monoxide Sensor, Measurement, 2010, 43, pp. $1115-1118$

[19] E. L. Hines, J. W. Gardner: An Artificial Neural Emulator for an Odor Sensor Array, Sensors and Actuators B, 18-19, 1994, pp. 661-664

[20] W. Ping, X. Jun: A Novel Recognition Method for Electronic Nose Using Artificial Neural Network and Fuzzy Recognition, Sensors and Actuators B 37, 1996, pp. 169-174

[21] H.-K. Hong, Ch. H. Kwon, S.-R. Kim, D. H. Yun, K. Lee, Y. K. Sung: Portable Electronic Nose System with Gas Sensor Array and Artificial Neural Network, Sensors and Actuators B 66, 2000, pp. 49-52

[22] D. Luo, H. G. Hosseini, J. R. Stewart: Application of ANN with Extracted Parameters from an Electronic Nose in Cigarette Brand Identification, Sensors and Actuators B 99, 2004, pp. 253-257 
[23] S. Osowski, T. H. Linh, K. Brudzewski: Neuro-Fuzzy Network for Flavor Recognition and Classification, IEEE Transactions on Instrumentation and Measurement, Vol. 53, No. 3, June 2004, pp. 638-644

[24] A. P. Singh, S. Kumar, T. S. Kamal: Virtual Compensator for Correcting the Disturbing Variable Effect in Transducers, Sensors and Actuators A 116, 2004, pp. 1-9

[25] T. Nenov, S. Ivanov: Linearization of Characteristic of Relative Humidity Sensor and Compensation of Temperature Impact, Sensors and Materials, 2007, Vol. 18, No. 2, pp. 95-106

[26] W. Hao, X. Li, M. Zhang: Application of RBF Neural Network to Temperature Compensation of Gas Sensor, Proceeding CSSE '08 Proceedings of the 2008 International Conference on Computer Science and Software Engineering - CSSE '08, Vol. 04, pp. 839-842 